\title{
Identification of a novel large CASR deletion in a patient with familial hypocalciuric hypercalcemia
}

\author{
Alejandro García-Castaño1,*, Leire Madariaga1,2,*, Sharona Azriel³, Gustavo Pérez de Nanclares ${ }^{1,2}$, \\ Idoia Martínez de LaPiscina', Rosa Martínez', Inés Urrutia', Aníbal Aguayo1,2, \\ Sonia Gaztambide1,2 and Luis Castaño1,2 \\ 'Biocruces Bizkaia Health Research Institute, CIBERDEM, CIBERER, Barakaldo, Spain, 2Hospital Universitario Cruces, \\ UPV/EHU, Barakaldo, Spain, and ${ }^{3}$ Hospital Infanta Sofia, Madrid, Spain
}

Correspondence should be addressed to L Castaño

Email

Icastano@osakidetza.eus

\section{Summary}

Familial hypocalciuric hypercalcemia type $I$ is an autosomal dominant disorder caused by heterozygous loss-of-function mutations in the CASR gene and is characterized by moderately elevated serum calcium concentrations, low urinary calcium excretion and inappropriately normal or mildly elevated parathyroid hormone (PTH) concentrations. We performed a clinical and genetic characterization of one patient suspected of familial hypocalciuric hypercalcemia type I. Patient presented persistent hypercalcemia with normal PTH and 25-hydroxyvitamin D levels. The CASR was screened for mutations by PCR followed by direct Sanger sequencing and, in order to detect large deletions or duplications, multiplex ligation-dependent probe amplification (MLPA) was used. One large deletion of 973 nucleotides in heterozygous state (c.1733-255_2450del) was detected. This is the first large deletion detected by the MLPA technique in the CASR gene.

\section{Learning points:}

- Molecular studies are important to confirm the differential diagnosis of FHH from primary hyperparathyroidism.

- Large deletions or duplications in the CASR gene can be detected by the MLPA technique.

- Understanding the functional impact of the mutations is critical for leading pharmacological research and could facilitate the therapy of patients.

\section{Background}

Familial hypocalciuric hypercalcemia type I (FHH1, OMIM \#145980) is an autosomal dominant disorder transmitted with a high degree of penetrance and characterized by moderately elevated serum calcium concentrations, low urinary calcium excretion and inappropriately normal or mildly elevated parathyroid hormone (PTH) concentrations. This disorder is caused by heterozygous loss-of-function mutations in the CASR gene (OMIM +601199) encoding the human calciumsensing receptor (CaSR).
Multiple disorders have been associated with defects in the CaSR as it plays a central role in the regulation of extracellular calcium homeostasis $(1,2)$. FHH1, neonatal hyperparathyroidism (NHPT, OMIM \#239200) and hypercalciuric hypercalcemia (3) are diseases caused by heterozygous loss-of-function mutations in the CASR gene, whereas activating mutations can result in isolated autosomal dominant hypocalcemia (OMIM \#601198) or combined with Bartter-like syndrome (OMIM \#601198). 
The aim of this study was to perform a clinical and genetic characterization of one patient suspected of familial hypocalciuric hypercalcemia. Patients with mutations of the CASR gene may not present such a classic picture of hypercalcemia with hypocalciuria or hypocalcemia with hypercalciuria. Molecular studies are important for confirming the diagnosis and distinguish it from other entities. One large deletion of 973 nucleotides in heterozygous state (c.1733255_2450del) was detected in our patient. Importantly, as far as we know, this is the first large deletion detected by multiplex ligation-dependent probe amplification (MLPA) technique in the CASR gene.

\section{Case presentation}

The study was approved by the Ethics Committee for Clinical Research of Euskadi (CEIC-E). The patient is a 56-year-old female of Philippine origin who was referred for evaluation of persistent hypercalcemia in several routine blood laboratory analyses. A history of hypercalcemia was reported in her family as well (her sister showed hypercalcemia and hyperparathyroidism, she does not have a genetic diagnosis yet). Unfortunately, we do not have any information about the parents. Laboratory results showed persistent hypercalcemia (serum calcium of $11.6 \mathrm{mg} / \mathrm{dL}$, reference range $8.5-$ 10.5 and total serum calcium corrected for albumin of $11.7 \mathrm{mg} / \mathrm{dL}$, reference range $8.6-10.2$ ) and serum phosphate at the lower limit of the normal range $(2.8 \mathrm{mg} /$ $\mathrm{dL}$, reference range 2.6-4.8), whereas intact PTH (40 pg/ $\mathrm{mL}$, reference range 10-65) and 25-hydroxyvitamin D levels (34.4 ng/mL, reference range 8.6-54.8) were within the normal range. The patient exhibited hypocalciuria. Specifically, her urinary calcium was very low (urinary calcium $<5 \mathrm{mg} / \mathrm{dL}$, reference range 0-250; urinary creatinine $35 \mathrm{mg} / \mathrm{dL}$, reference range 90-300) despite their high serum calcium levels, and she had a $24-\mathrm{h}$ urine calcium:creatinine clearance ratio (CCCR) $<0.01$. Therefore, the patient was suspected of FHH and the CASR gene genetics analysis was requested. Four years previously, she had developed hyperthyroidism due to Graves-Basedow disease, and she had been treated with antithyroid medications and radioiodine therapy. Furthermore, the patient presented other personal history as hypercholesterolemia, prediabetes and femoral and lumbar osteoporosis (markers of bone remodeling: serum alkaline phosphatase $60 \mathrm{U} / \mathrm{L}$, reference range $46-116 \mathrm{U} / \mathrm{L}$; serum osteocalcin $3.02 \mathrm{ng} / \mathrm{mL}$, reference range $\quad 0-22 ; \quad \beta$-isomerized C-telopeptides $0.12 \mu \mathrm{g} / \mathrm{L}$, reference range $0-0.84$ ).

\section{Investigation}

In order to detect large deletions or duplications in the CASR region, a commercially available MLPA kit, SALSA MLPA probemix P177-B2 (MRC Holland, Amsterdam, The Netherlands), was used. This MLPA kit contains 14 MLPA probes specific for the CASR gene with amplification products between 130 and 301 nucleotides. In addition, it contains nine reference probes expected to have a normal copy number that we used for the intrasample normalization, and nine internal quality control fragments generating an amplification product smaller than 120 nucleotides.

The exons and flanking intronic sequences of the CASR gene (Ensembl identifiers: gene ENSG00000036828; transcript, ENST00000639785.1) were screened for mutations by PCR followed by direct sequencing. We used primers to amplify exons 2-7 together with their splice sites. Furthermore, we designed primers to amplify a large fragment where the large deletion, found by the MLPA technique, is included (Forward: 5'-ACAGGTAAGGGAACCCCTCTT-3'; Reverse: 5'GCCTCCACCACTGATGACAA-3'). Purified amplified products were directly sequenced in both directions with fluorescent dideoxynucleotides (BigDye Terminator v3.1 Cycle Sequencing Kit, Life Technologies). DNA mutation was named according to the Human Genome Variation Society guidelines (www.hgvs.org).

With respect to genotype, one large deletion of 973 nucleotides in heterozygous state (marked in bold in the CASR sequence showed in Table 1) was detected by the MLPA technique (Fig. 1). The amplification of exon 7 using specific primers that jointed to the deleted region confirmed the heterozygous state in the patient. Subsequently, to delimit the deletion, we amplified one fragment of 2926 nucleotides by long-PCR and delimited the deletion to 973 nucleotides by sequencing with internal primers. The deletion expands from nucleotide in c.1733-255 position within intron 6 to nucleotide c.2518 within exon 7 (c.1733-255_2450del). This deletion is supposed to generate a protein shorter than WT, as the splice site of exon 7 is affected. Therefore, the mutant CaSR protein probably loses the transmembrane domain and the intracellular carboxyl-terminal tail (amino acids 578 to 1078 ).

As far as we know, this is the first large deletion detected by MLPA technique in the CASR gene. 


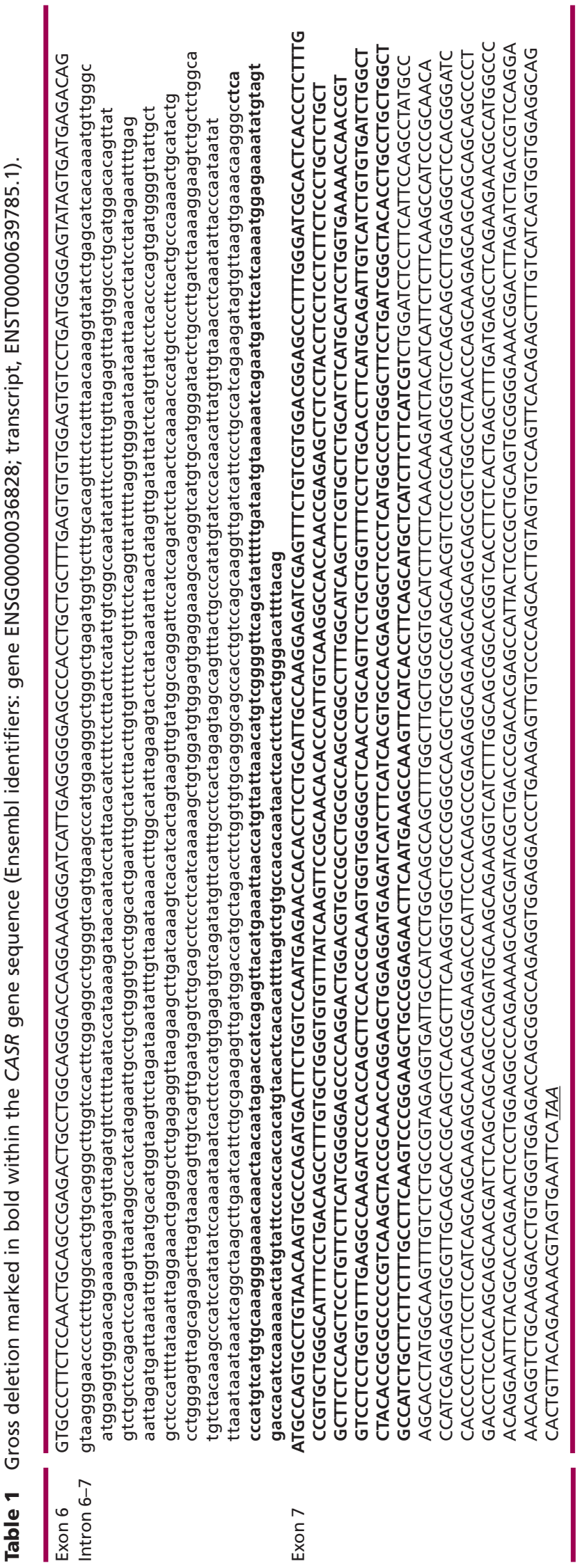

\section{Discussion}

In this study, we have described the first large deletion found in the CASR gene by the MLPA technique. Structural analysis of CASR gene confirmed that the patient presented a deletion of 973 nucleotides in heterozygous state (c.1733-255_2450del). Patient had been previously diagnosed with $\mathrm{FHH}$, and this deletion affects the splice site of exon 7 in a gene where loss of function is a known mechanism of disease; consequently, this large deletion has very strong evidence of pathogenicity; therefore, it has been classified as PVS1-null variant (4).

It has been hypothesized that some punctual mutations exert a dominant negative effect in heterozygous state, interfering with the WT receptor signal and, as a result, produce a more severe biochemical phenotype (5). As the splice site of exon 7 is included in the deleted region, it is supposed that the mutant protein lacks both the transmembrane and the intracellular domain. Thus, the allele presenting the deletion produces a short protein that is retained in the cytoplasm, as other studies have demonstrated with nonsense mutations (6). We hypothesized that our patient only presents CaSR receptors codified by the allele without the deletion in the membrane, as the mutant protein is probably unable to anchor the cell membrane and is presumed to be non-functional and thus, does not interfere with the WT receptor signal. Therefore, patient presents a less severe phenotype as observed in others patients with nonsense mutations of the CASR gene (6). Nevertheless, we recognized the need to conduct functional analysis to confirm the pathogenicity of our novel mutation.

In addition, we found the p.Arg990Gly polymorphism in the allele without the deletion. Some authors have reported that patients with this polymorphism and primary or secondary hyperparathyroidism have lower levels of serum PTH and ionized calcium (7), as shown in our patient. However, the influence of this polymorphism on the patient's phenotype is not clear, as it is very common in East Asia (0.50, according to ExAC, https:// exac.broadinstitute.org).

Up to date, only two gross deletions have been described in the literature. The first one was an activating mutation which consisted in a large deletion of 181 amino acids in the carboxyl-terminal tail (Ser895-V1075) (8). Interestingly, patient with this deletion presented autosomal dominant hypocalcemia. Authors suggested that the mutant CaSR exerts a dominant positive effect in the membrane. Moreover, it was observed more defective CaSR in the membrane in heterozygous patients 
A
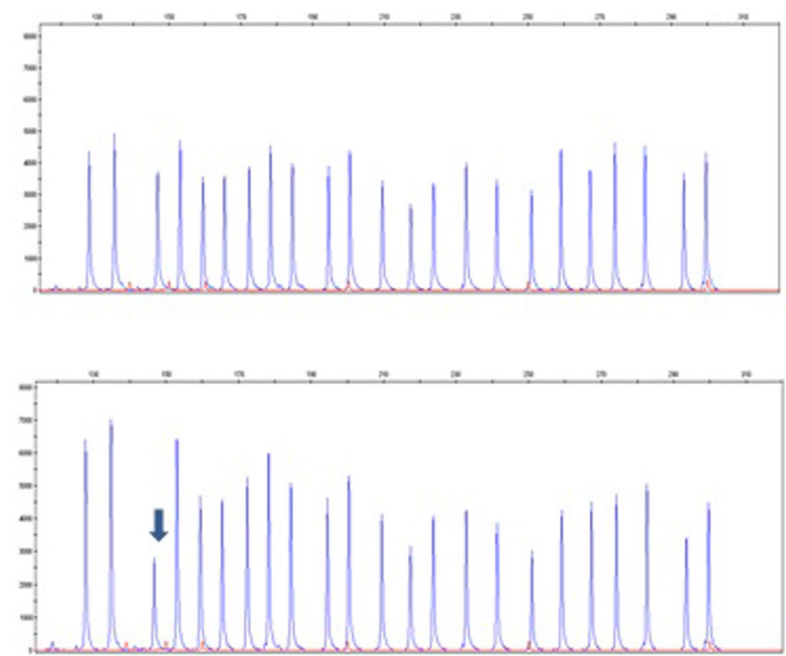

B

Reference Sequence

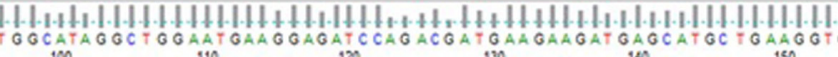

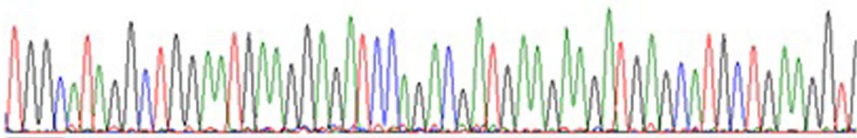

Deletion

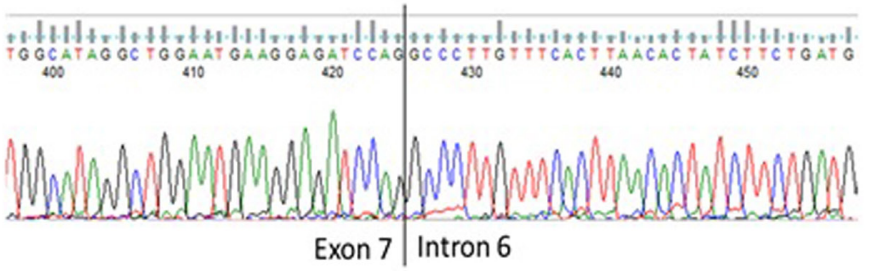

Figure 1

(A) Detection of the CASR deletion by the MLPA techniques. MLPA electropherogram for the CASR gene from control (upper panel) and patient (lower panel). Each peak represents the exons for the CASR gene and nine reference probes. The arrow shows half dose for probe 3 corresponding to the beginning of Exon 7. (B) Novel CASR deletion detected by direct sequencing. Figure represents the sequencing chromatograms from control (upper panel) and patient (lower panels). Hemizygous c.1733-255_2450del mutation; vertical line shows the beginning of the deletion.

presenting the deletion. The second deletion consisted in a large deletion of chromosome 3p13.31-22.1 that included 101 known genes, including the CASR gene. The individual presented multiple disorders, including hypercalcemia and hypocalciuria (9).

Identification of large deletions by MLPA is remarkably rare in the CASR gene. Thus, we only identified one copy number variation in 56 patients $(2 \%)$ with calcium metabolism disorders using the MLPA technique (data not published). Moreover, a previous study did not identify any copy number variation in a large cohort of 257 patient samples using MLPA $(9,10)$. In our laboratory, firstly, the exons and flanking intronic sequences of the CASR gene are screened for mutations by PCR followed by direct sequencing, and secondly, when the results are negative, the MLPA is performed. This technique is a useful tool to check copy number variations (CNVs). In our experience, the next-generation sequencing shows great sensitivity but little specificity in CNVs detection. Therefore, we propose to first look for mutations by sequencing, should mutation not be detected, MLPA or QMPSF (quantitative multiplex PCR of short fluorescent) analyses must be carried out in order to complete the molecular study.

In conclusion, molecular studies are important to confirm the differential diagnosis of FHH from primary hyperparathyroidism (thus preventing surgery) and other diseases, because of common biochemical features. Understanding the functional impact of the mutations at the cell membrane is critical for leading pharmacological research and could facilitate the therapy of patients. Moreover, performing genetic analysis is essential to detect carriers, because some individuals with mutations remain normocalcemic. The aim of this study was to describe the first gross deletion found using the MLPA technique. Punctual mutations are the most common variants described in the CASR gene (according to the Human Gene Mutation Database, 348 mutations). We demonstrated that MLPA is a useful technique for the detection of large deletions or duplications in the CASR gene. Therefore, we recommended the use of this technique to complete the molecular study.

Declaration of interest

The authors declare that there is no conflict of interest that could be perceived as prejudicing the impartiality of this case report.

\section{Funding}

This study was supported by a grant from the Department of Health of the Basque Government (2014111064) and the Department of Education of the Basque Government (IT795-13). The funders had no role in study design, data collection and analysis, decision to publish or preparation of the manuscript.

\section{Patient consent}

The patient provided written, informed consent to participate in this study. The information has been sufficiently anonymized, making it impossible to identify the patient with any certainty. 


\section{Author contribution statement}

Writing: A G-C, L M and L C. Study design: A G-C, L M, S A, S G and L C. Study conduct: A G-C, L M, G P d N, A A and L C. Data collection: S A, A A. Data analysis: A G-C and G P d N. Data interpretation: A G-C, G P d N, I M d L, I U and R M. Revising manuscript content: S A, I M d L, S G and L C.

\section{Acknowledgements}

The authors thank the patient and their endocrinologist who collaborated with the genetic study.

\section{References}

1 Aida K, Koishi S, Tawata M \& Onaya T. Molecular cloning of a putative $\mathrm{Ca}(2+)$-sensing receptor cDNA from human kidney. Biochemical and Biophysical Research Communications 1995214 524-529. (https://doi.org/10.1006/bbrc.1995.2318)

2 Brown EM, Gamba G, Riccardi D, Lombardi M, Butters R, Kifor O, Sun A, Hediger MA, Lytton J \& Hebert SC. Cloning and characterization of an extracellular $\mathrm{Ca}(2+)$-sensing receptor from bovine parathyroid. Nature 1993366 575-580. (https://doi. org/10.1038/366575a0)

3 Carling T, Szabo E, Bai M, Ridefelt P, Westin G, Gustavsson P, Trivedi S, Hellman P, Brown EM, Dahl N, et al. Familial hypercalcemia and hypercalciuria caused by a novel mutation in the cytoplasmic tail of the calcium receptor. Journal of Clinical Endocrinology and Metabolism 200085 2042-2047. (https://doi. org/10.1210/jcem.85.5.6477)

4 Richards S, Aziz N, Bale S, Bick D, Das S, Gastier-Foster J, Grody WW, Hegde M, Lyon E, Spector E, et al. Standards and guidelines for the interpretation of sequence variants: a joint consensus recommendation of the American College of Medical Genetics and
Genomics and the Association for Molecular Pathology. Genetics in Medicine 201517 405-424. (https://doi.org/10.1038/gim.2015.30)

5 Ward BK, Magno AL, Blitvich BJ, Rea AJ, Stuckey BG, Walsh JP \& Ratajczak T. Novel mutations in the calcium-sensing receptor gene associated with biochemical and functional differences in familial hypocalciuric hypercalcaemia. Clinical Endocrinology 200664 580-587. (https://doi.org/10.1111/j.1365-2265.2006.02512.x)

6 Ward BK, Magno AL, Davis EA, Hanyaloglu AC, Stuckey BG, Burrows M, Eidne KA, Charles AK \& Ratajczak T. Functional deletion of the calcium-sensing receptor in a case of neonatal severe hyperparathyroidism. Journal of Clinical Endocrinology and Metabolism 200489 3721-3730. (https://doi.org/10.1210/jc.2003-031653)

7 Terranegra A, Ferraretto A, Dogliotti E, Scarpellini M, Corbetta S, Barbieri AM, Spada A, Arcidiacono T, Rainone F, Aloia A, et al. Calcimimetic R-568 effects on activity of R990G polymorphism of calcium-sensing receptor. Journal of Molecular Endocrinology 201045 245-256. (https://doi.org/10.1677/JME-10-0034)

8 Lienhardt A, Garabédian M, Bai M, Sinding C, Zhang Z, Lagarde JP, Boulesteix J, Rigaud M, Brown EM \& Kottler ML. A large homozygous or heterozygous in-frame deletion within the calciumsensing receptor's carboxylterminal cytoplasmic tail that causes autosomal dominant hypocalcemia. Journal of Clinical Endocrinology and Metabolism 200085 1695-1702. (https://doi.org/10.1210/ jcem.85.4.6570)

9 Materna-Kiryluk A, Kiryluk K, Burgess KE, Bieleninik A, SannaCherchi S, Gharavi AG \& Latos-Bielenska A. The emerging role of genomics in the diagnosis and workup of congenital urinary tract defects: a novel deletion syndrome on chromosome 3q13.31-22.1. Pediatric Nephrology 201429 257-267. (https://doi.org/10.1007/ s00467-013-2625-2)

10 Nissen PH, Christensen SE, Wallace A, Heickendorff L, Brixen K \& Mosekilde L. Multiplex ligation-dependent probe amplification (MLPA) screening for exon copy number variation in the calcium sensing receptor gene: no large rearrangements identified in patients with calcium metabolic disorders. Clinical Endocrinology 201072 758-762. (https://doi.org/10.1111/j.1365-2265.2009.03750.x)

Received in final form 22 October 2018

Accepted 14 November 2018 